\title{
Design of LEO Constellations Providing Internet Services Based on SOC Method
}

\author{
Quan Chen ${ }^{1, a}$, Yuzhu Bai ${ }^{1}$, Lihu Chen ${ }^{1}$ and Ziyan Pang ${ }^{2}$ \\ ${ }^{1}$ College of Aerospace Science and Engineering, National University of Defense Technology, Changsha 410072, \\ China \\ ${ }^{2}$ College of Basic Education for Commanding Officers, National University of Defense Technology, Changsha \\ 410072, China
}

\begin{abstract}
Narrow-beamwidth antenna is applied to LEO satellite constellations to provide high-speed internet services for the globe. Low-altitude, limited coverage region of single satellite and large scale are the main features of them. In this paper, under the constraint of specified half-beam angle of antenna, both inclined and polar orbit constellations are analysed based on Streets-of-Coverage method to provide continuous coverage. The results indicate that the coverage performance of single satellite is the key factor which can affect the configuration and number of constellations. When the orbit altitude is $1200 \mathrm{~km}$ and half-beam angle is 32 degrees, up to thousands of satellites are required for constellations providing internet services for zonal region 60 degrees from the equator. Non-symmetrical polar constellations show the best performance of continuous coverage and require approximately half of the satellites of inclined orbit constellations. The required satellites with different single coverage performance are also investigated as a reference for future research.
\end{abstract}

\section{Introduction}

A major trend of satellite development is a decrease of satellite size and cost. Instead of traditional single larger satellite, distributed micro-satellite systems are widely deployed to implement complex missions [1]. This has led to the rapid development of constellations with a large number of satellites. OneWeb [2] has announced a constellation consisting of up to 648 satellites to provide high-speed internet services. Meanwhile, SpaceX is planning to launch more than 4000 satellites to build a gigantic space-based internet communication system [3].

Satellite with a higher altitude usually has a better performance in coverage, but the corresponding attenuation of space link is greater, which is not suitable for high-speed broadband signal transmission. Generally, the optimal orbital altitude for LEO satellites ranges from 500 to $1500 \mathrm{~km}$. Elliptical-orbit satellites are able to stay for a longer time above certain hemisphere. However, the variation of altitude brings about a higher requirement for the on-board components when satellites fly across the Van Allen radiation belt. Circular low earth orbit consequently becomes the most popularly used in communication constellations [4].

For satellite systems providing internet services, a large zonal or the global region should be continuously covered to ensure the availability at any time and any place. Traditional methods and

\footnotetext{
${ }^{a}$ Corresponding author : chenquan11@foxmail.com
} 
results are based on the constraint of a lower minimum elevation angle because the coverage region of single satellite is large enough [5]. However, to transmit high-speed and broadband signals, satellites are equipped with narrow-beamwidth antennas which limit the coverage of single satellite [6]. Then the satellite amount can be extremely large such as OneWeb and SpaceX satellite constellations.

In this paper, the half-beam angle of antenna is given as the primary constraint, and Streets-ofCoverage method [7] is applied to design the optimal constellation providing continuous coverage of the mid-and low-latitude zonal regions. The influence of inclination and the number of orbital planes is discussed. Both inclined and polar satellite constellations are analyzed and compared so as to find out the optimal results. Finally the results of required satellite number at different altitudes are listed.

\section{Single coverage}

The coverage region of single satellite, illustrated by Figure 1, is defined as the spherical surface formed by the intersection of the satellite-centered-cone and the earth. Minimum elevation angle is the common constraint in traditional analysis, and a point is covered by a satellite if the local elevation angle is greater than the specified minimum value. However, in terms of LEO satellites offering highspeed data communication service, $\mathrm{Ku}, \mathrm{Ka}$ or even higher frequency is used which leads to greater attenuation of space link [6,8]. Then high-gain antenna is on demand to compensate for the attenuation. The relation of the antenna gain and beamwidth is given by

$$
G=\eta\left(\frac{\pi \times 70}{\alpha_{0.5}}\right)^{2}
$$

where $G$ is the antenna gain, $\alpha_{0.5}$ is the half power beamwidth in degree and $\eta$ is the antenna efficiency. It is obvious that reducing beamwidth is an effective approach to improve the gain. Thus narrow-beamwidth antenna is designed to transmit high-speed signals in satellite-ground links. Although multi-beam antenna is adopted to enlarge the whole beamwidth, the coverage region is much smaller than traditional communication satellites or observation satellites. In this situation, beam-angle becomes the key factor in the design of satellite constellations providing internet services.

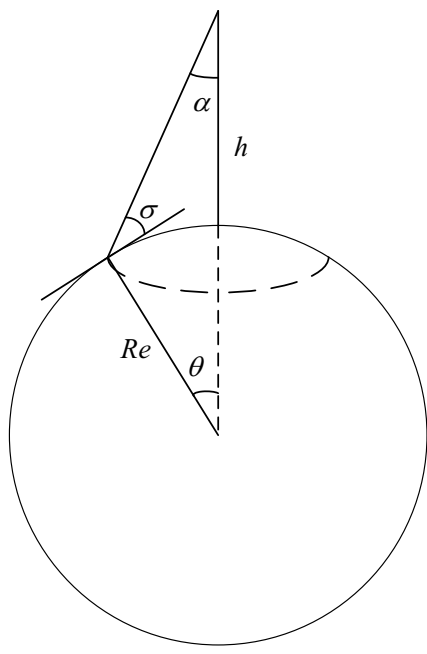

Figure 1. Coverage geometry.

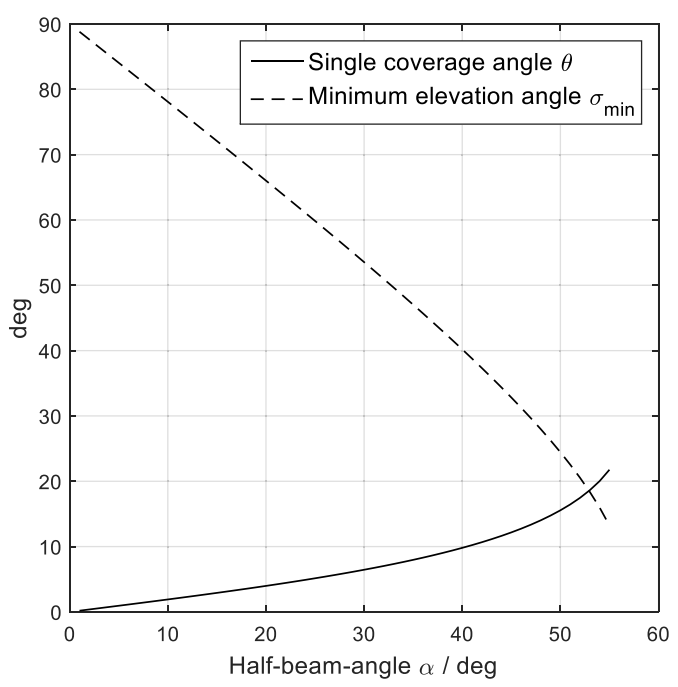

Figure 2. Coverage angle and minimum elevation angle versus half-beam angle.

A satellite with altitude $h$ and half-beam angle $\alpha$ is able to cover a spherical segment of the earth's surface. The geocentric half-cone-angle of the coverage region is defined as coverage angle $\theta$ in this paper. From the geometry, it follows that 


$$
\theta=\arcsin \left(\frac{h+\mathrm{Re}}{\mathrm{Re}} \sin \alpha-\alpha\right)
$$

where $\mathrm{Re}$ is the radius of the earth. When the satellite altitude is fixed, following equation shows the relation between half-beam angle $\alpha$ and minimum elevation angle $\sigma_{\min }$ of the coverage region.

$$
\sigma_{\min }=\arccos \left(\frac{h+\operatorname{Re}}{\operatorname{Re}} \sin \alpha\right)
$$

Figure 2 shows the relation between $\theta, \sigma_{\min }$ and $\alpha$, when the satellite altitude is fixed $(h=1200 \mathrm{~km})$. According to the curves, with the increase of half-beam angle, the single coverage region reduces while the minimum elevation angle of the coverage region increases gradually. In the case of $\alpha=30^{\circ}$, corresponding angles are $\theta=6.45^{\circ}$ and $\sigma_{\min }=53.55^{\circ}$.

\section{Constellation design method based on SOC}

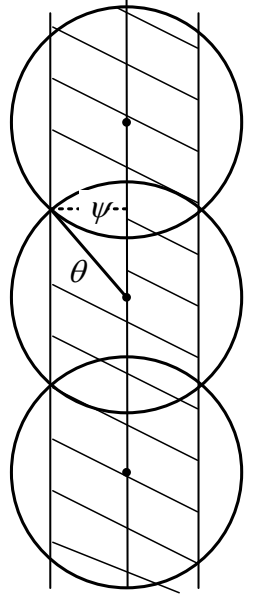

Figure 3. Streets of coverage.

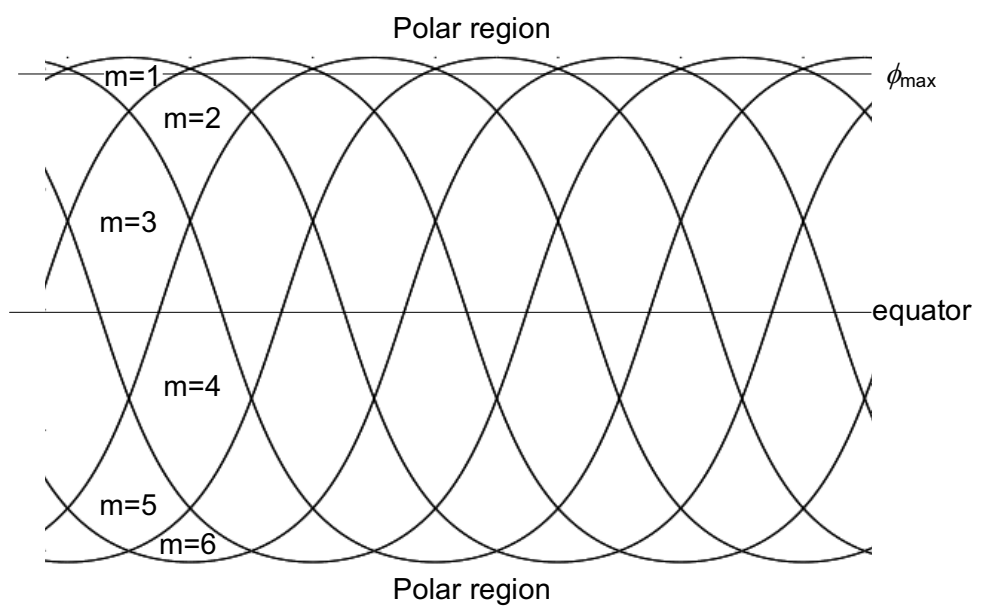

Figure 4. Geometry of the mesh $\left(n_{1}=7\right)$.

\subsection{Streets-of-Coverage methods}

Streets-of-Coverage (SOC) method solves the problem of continuous coverage for a zonal or global region using the overlapped coverage regions of adjacent intra-plane satellites which can be continuously viewed. In SOC method, constellation configuration shows following features: 1) All of the orbits are circular with the same altitude and inclination. 2) The constellation consisting of $n_{1}$ orbit planes and each contains $n_{2}$ satellites. 3) Satellites in each plane are evenly distributed.

It can be seen from Figure 3, when single coverage angle $\theta$ satisfies $\theta>\pi / n_{2}$, streets viewed continuously are formed by overlapping coverage regions of adjacent intra-plane satellites. By means of spherical trigonometry, the street width follows

$$
\cos \theta=\cos \psi \cos \left(\pi / n_{2}\right)
$$

In SOC method, only streets covered continuously are considered while the coverage beyond the streets is neglected. Consequently, the phase difference between adjacent plane satellites can be eliminated and the circular coverage regions are simplified as strips corresponding to each plane. Then the goal of the constellation design is to calculate the required street width of each constellation configuration so that the specified zonal or global region can be completely covered [7]. 


\subsection{Method for inclined orbit constellation}

Considering the zonal region to be continuously covered that ranges from the equator to latitude $\phi_{\max }$, the orbit inclination is $i$, and $n_{1}$ orbital planes are evenly distributed along the equator. By projecting the orbit trace at certain moment, the earth's surface can be divided into $n_{1}-1$ layer meshes and two polar regions, as Figure 4 shows. The latitude of the intersections from north to south is

$$
\phi_{j}=\arctan \left[\tan i \cos \left(j \pi / n_{1}\right)\right]
$$

where $j=1, \ldots, n_{1}-1$ and $-i<\phi_{j}<+i$. Then define $\phi_{0}=i$ and $\phi_{n}=-i$. Note that $\phi_{1}$ is the latitude of the northernmost grid node. If $\phi_{\max } \leq \phi_{1}$, the only coverage of inside mesh regions needs to be considered. Figure 5 gives a general geometry of $m$ th-layer mesh. Point $P$ is the center of the inscribed circle that is tangent to all the four boundaries of the mesh. $X_{U}$ is the angle from $P$ to the upper vertex. Let $\psi_{\min , m}$ be the required minimum width of streets to cover the $m$ th-layer mesh and it is also the angle between $P$ and either of the boundaries. $\psi_{\min , m}$ can be calculated by

$$
\sin \psi_{\min , m}=\sin X_{U} \cos i / \cos \phi_{m-1}
$$

where

$$
X_{U}=\arctan \left\{\sin \left(\frac{m \pi}{n_{1}}\right) \sin \left(\frac{\pi}{n_{1}}\right) \tan i\left\{1+\cos \left(\frac{m \pi}{n_{1}}\right) \cos \left(\frac{\pi}{n_{1}}\right) \cos \left[(m-1) \frac{\pi}{n_{1}}\right] \tan ^{2} i\right\}^{-1}\right\}
$$

and the overall required width of streets $\psi_{\min }$ is the maximum value of $\psi_{\min , m}$ for meshes needed to be covered.

If $\phi_{\max }>\phi_{1}$, additional coverage outside the $n_{1}-1$ layer meshes should be considered as well, as Figure 6 illustrates. The required street width to cover the external region is given by

$$
\psi_{\text {ext }}=\arcsin \left[\sin \phi_{\max } \cos i-\cos \phi_{\max } \sin i \cos \left(\pi / n_{1}\right)\right]
$$

In this situation, the required minimum width is

$$
\psi_{\min }=\max \left\{\psi_{\min , 1}, \psi_{\min , 2}, \ldots, \psi_{\min , n_{1}-1}, \psi_{\text {ext }}\right\}
$$

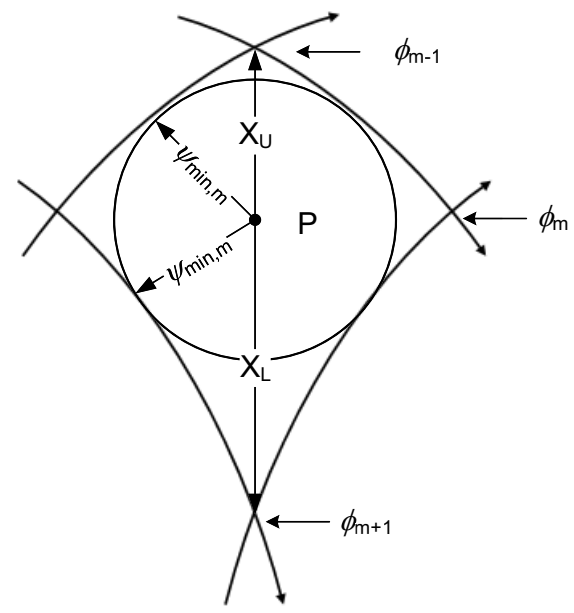

Figure 5. General geometry of $m$ th-layer mesh.

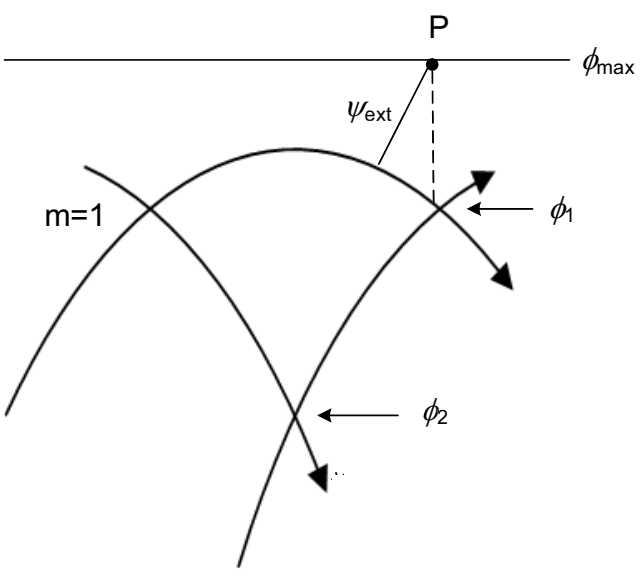

Figure 6. Coverage for regions beyond the mesh.

When the orbit altitude $h$ and half-beam angle $\alpha$ are given, the coverage angle $\theta=\theta(h, \alpha)$ can be solved from equation (2). If $\theta \leq \psi_{\min }$, i.e. the coverage angle of single satellite is no greater than the 
needed minimum street width, then it is impossible to form the streets with required width. When $\theta>\psi_{\min }$, combining $\psi>\psi_{\min }$ and equation (4) yields the required number of satellite per plane.

$$
n_{2} \geq \frac{\pi}{\arccos \left(\cos \theta / \cos \psi_{\text {min }}\right)}
$$

Then the required minimum number of the satellite $N$ can be obtained by $N=n_{1} n_{2}$

$$
N=n_{1}\left\lceil\frac{\pi}{\arccos \left(\cos \theta / \cos \psi_{\text {min }}\right)}\right\rceil, \quad \theta>\psi_{\text {min }}
$$

Note that if the inclination is much smaller than $\phi_{\max }$, the value of $\psi_{\text {ext }}$ will be the crucial factor with a large value. If the performance of single coverage is poor, then the constraint $\theta>\psi_{\min }$ will not be satisfied at any $n_{1}$. In addition, $\psi_{\min }$ will be great at a relatively small $n_{1}$, which makes it impossible to form streets with required width. Consequently, both $i$ and $n_{1}$ should be greater than certain values to ensure the existence of a reasonable solution. Limitation of $i$ and $n_{1}$ can be obtained by iteratively calculating $N$ through different $i$ and $n_{1}$. The solution procedures are listed in Table 1 .

Table 1. Solution procedures of inclined orbit constellations.

Step 1. Calculate the single coverage angle $\theta$.

Step 2. Set the number of orbital planes $n_{1}$ and inclination $i$.

Step 3. Calculate required minimum street width of each candidate mesh region $\psi_{\min , m}$ and $\psi_{\text {ext }}$.

Step 4. Solve the overall $\psi_{\min }$.

Step 5.

Compare $\psi_{\min }$ with $\theta$. If $\theta \leq \psi_{\min }$, no solution for given $\left(n_{1}, i\right)$ set, go to Step 2; if $\theta>\psi_{\min }$, go to Step 6.

Step 6. Calculate the required number of satellites per plane $n_{2}$.

Step 7. Obtain the minimum total number of satellite $N$ of given $\left(n_{1}, i\right)$ set.

\subsection{Method for polar constellation}

\subsubsection{Symmetrical polar constellation}

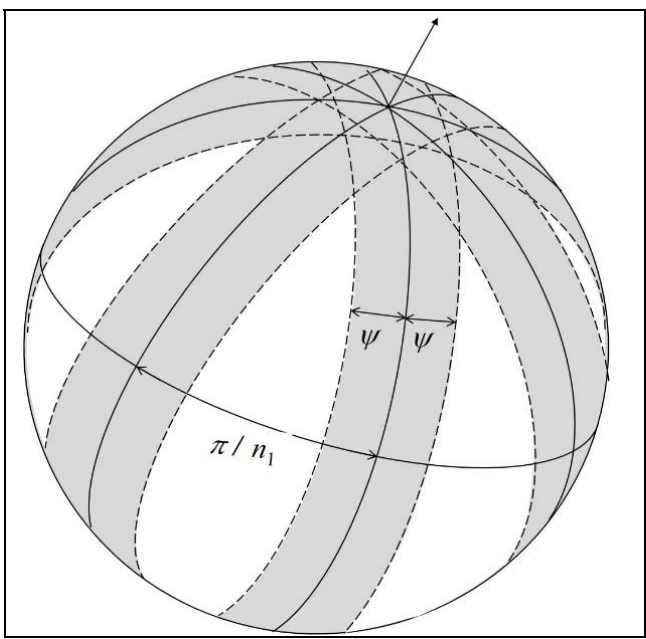

Figure 7. Symmetrical polar constellation. 
The inclination of polar satellites is $90^{\circ}$ and generally the ascending nodes spread within $(0, \pi)$ to fully utilize the coverage of ascending and descending orbits. For symmetrical polar constellation, the $n_{1}$ planes are uniformly separated by $\Delta$, where $\Delta=\pi / n_{1}$, as is illustrated in Figure 7 .

Since all the orbits of polar constellation intersect at two poles, polar regions are the most likely ones to be covered while lower-latitude regions are more difficult. Continuous global coverage can be achieved once the equator is continuously covered. Consequently, to cover a zonal region including the equator, the required minimum street width should be

$$
\psi_{\min }=\frac{\pi}{2 n_{1}}
$$

The following steps are similar to those of inclined orbit constellation design. After given the orbit altitude $h$ and half-beam angle $\alpha$, one can obtain the coverage angle $\theta=\theta(h, \alpha)$. The combination of equations (4), (10), (11) and (12) yields the required number of satellites $N$ in a symmetrical polar constellation to cover the entire globe.

$$
N=n_{1}\left\lceil\frac{\pi}{\arccos \left(\cos \theta / \cos \left(\pi / 2 n_{1}\right)\right)}\right\rceil, \quad \theta>\frac{\pi}{2 n_{1}}
$$

\subsubsection{Non-symmetrical polar constellation}

The coverage beyond the street in the above-mentioned constellations is not considered, which wastes the extra coverage between two planes. For polar constellations, there are two kinds of interface of two adjacent orbits, i.e. co-rotating interface and counter-rotating interface. In co-rotating interface, the relative positions of adjacent satellites are fixed and the phase separation between satellites in adjacent orbits can be arranged so that the largest continuously covered regions can be generated through the optimal phase shift. However, since the rapid variation of relative positions in counterrotating interfaces, adjacent orbits should be close enough to form a continuous Streets-of-Coverage. Therefore, the orbital planes can be asymmetrically arranged to make full use of the coverage regions.
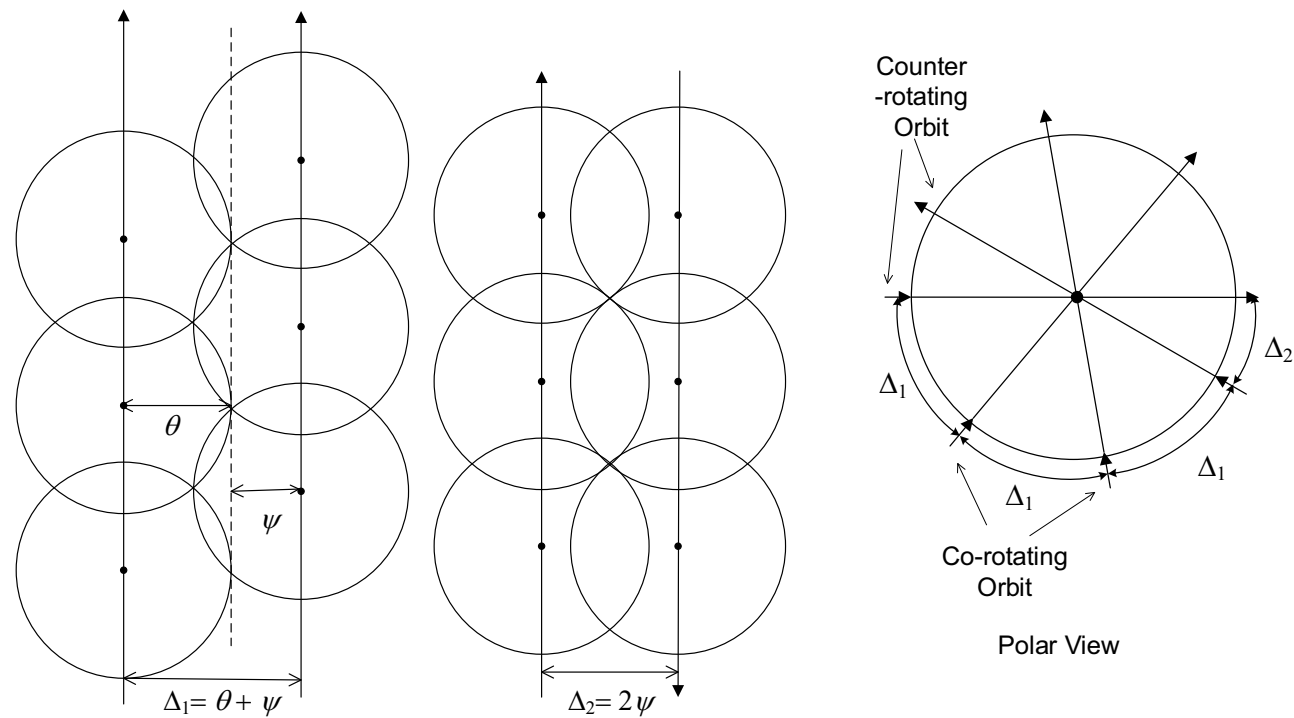

Figure 8. Co- and counter-rotating orbits and the seam of non-symmetrical polar constellation.

According to Figure 8, the angular separations of co-rotating orbits are

$$
\Delta_{1}=\theta+\psi
$$

and those of counter-rotating orbits are 


$$
\Delta_{2}=2 \psi
$$

For the polar constellation with $n_{1}$ planes, it contains $2\left(n_{1}-1\right)$ co-rotating interfaces and two counter-rotating interfaces. The relation between them is given by

$$
\left(n_{1}-1\right) \Delta_{1}+\Delta_{2}=\pi
$$

When the single coverage angle and number of orbit planes are given, the required minimum street width can be calculated by combining equation (14), (15),(16) and $\theta>\psi_{\min }$

$$
\psi_{\text {min }}=\frac{\pi-\left(n_{1}-1\right) \theta}{n_{1}+1}, \quad n_{1}>\frac{\pi}{2 \theta}
$$

Note that in this step, the single coverage angle $\theta$ should be known. Then the required satellites can be obtained through equation (11).

\section{Case study}

\subsection{Inclined orbit constellation case}

Based on the constraint of the on-board payload, the satellite altitude and half-beam angle are given ( $h=1200 \mathrm{~km}, \alpha=32^{\circ}$ ). Considering the distribution characteristics of global population and economic activities, zonal region 60 degrees from the equator is required to be continuously covered (i.e. $\left.\phi_{\max }=60^{\circ}\right)$. The required minimum street width $\psi_{\min }$ and number of satellites $N$ at different inclinations are analyzed.

Figure 9 illustrates that the required street width gradually decreases with the increase of the number of orbital planes $n_{1}$. Comparison between curves of $60^{\circ}$ and $70^{\circ}$ inclination shows that constellations with larger inclination require more satellites. The curve of $50^{\circ}$ inclination decreases rapidly when the number of planes is less than 15 and then remains stable.

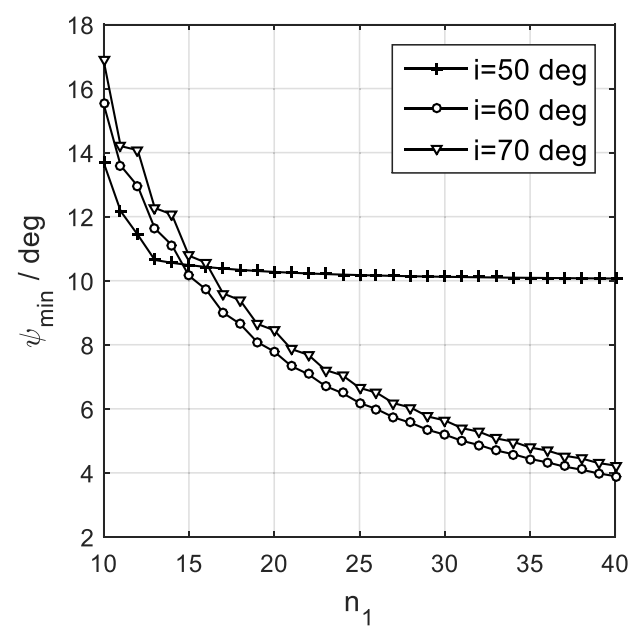

Figure 9. Required street width at different inclinations.

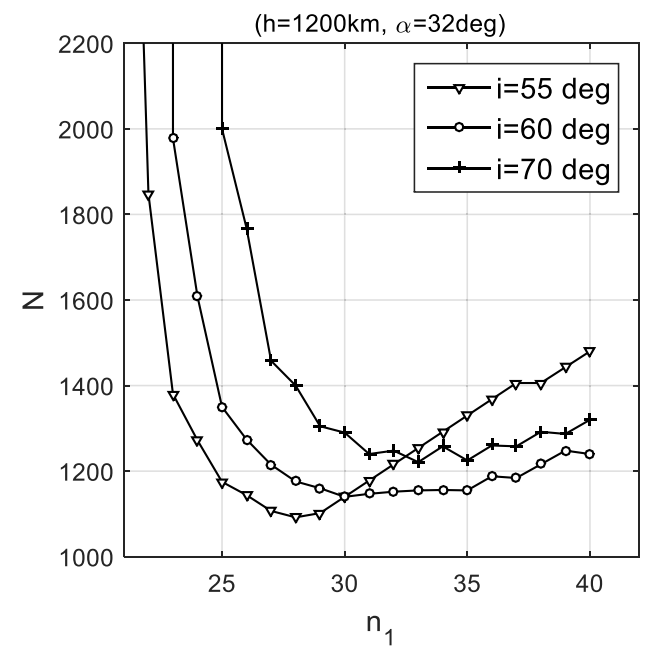

Figure 10. Satellite number of inclined orbit constellations.

When $h=1200 \mathrm{~km}, \alpha=32^{\circ}$, the coverage angle $\theta=7.03^{\circ}$ can be solved through equation(2). By iterating through the available combinations of $n_{1}$ and $i$, it is found that the orbit inclination should be greater than $53.1^{\circ}$ for $\theta>\psi_{\min }$. By means of the foregoing method in Table 1, the required satellite amount $N$ affected by $n_{1}$ can be obtained, as is displayed in Figure 10 . When $n_{1}$ is much small, the 
given single coverage is unable to form the required large $\psi_{\min }$. In this case, when $i=55^{\circ}$, it is found that $n_{1}$ should be greater than 21 through iterative calculations. $N$ is directly determined by $n_{1}$ and $n_{2}$. And the increase of $n_{1}$ will induce smaller $\psi_{\min }$ and then lower the following $n_{2}$. Thus there is an optimal $n_{1}$ for constellations with minimum satellites. By calculating required number of satellites $N$ at each set of $n_{1}$ and $i$ repeatedly, one can obtain the relations among them. Figure 10 shows that the required number of satellites has a rapid decrease firstly and then grows with the increase of planes. The least number of satellites exists when the optimal value of $n_{1}$ is close to 30 . The optimal constellations at different inclinations are investigated and the optimal results are listed in Table 2. It can be seen that when the inclination is $55.1^{\circ}$, it requires the least number of satellites 1073 in 29 planes.

Table 2. Optimal inclined orbit constellations with different inclinations for continuously coverage (at $h=1200 \mathrm{~km}, \alpha=32 \mathrm{deg}, \phi_{\max }=60 \mathrm{deg}$ ).

\begin{tabular}{ccccl}
\hline$i /$ deg & $N$ & $n_{1}$ & $n_{2}$ & $\min / \operatorname{deg}$ \\
\hline 55 & 1092 & 28 & 39 & 5.26 \\
60 & 1140 & 30 & 38 & 5.19 \\
70 & 1221 & 33 & 37 & 5.08 \\
80 & 1254 & 33 & 38 & 5.18 \\
55.1 & 1073 & 29 & 37 & 5.06 \\
\hline
\end{tabular}

\subsection{Polar constellation case}

To continuously cover the same zonal region as in previous case, polar constellations should be able to continuously cover the equator, i.e. the entire globe. The influence of the number of orbital planes $n_{1}$ on satellites number $N$ is obtained when the coverage performance of single satellite is the same as that in inclined constellation case. In Figure 11, similar to inclined orbit constellation, $N$ decreases with the growth of $n_{1}$ and then increases in both symmetrical and non-symmetrical cases. The optimal result of symmetrical polar constellation is 663 satellites in 17 orbital planes while 522 satellites in 18 planes in non-symmetrical case.

Table 3 lists the optimal results of different constellation configurations. It shows that the required satellites are substantially cut down compared to inclined cases under the same single coverage performance. The first two results indicate that polar constellation requires less orbital planes while offering a wider Streets-of-Coverage than inclined orbit constellation. Therefore, in polar constellation, the overlapped regions between adjacent planes are smaller, which manifests a higher efficiency of single coverage utilization. In addition, non-symmetrical polar configuration is better than symmetrical configuration since phase arrangement enlarges the continuous coverage region and thus reduces the number of satellites per orbital plane. Note that $\psi_{\min }$ of non-symmetrical case in the table is the required streets width in counter-rotating interface.

Table 3. Optimal constellation results of different configurations

\begin{tabular}{ccccc|c}
\multicolumn{6}{c}{ (at $\left.h=1200 \mathrm{~km}, \alpha=32 \mathrm{deg}, \phi_{\max }=60 \mathrm{deg}\right)}$. \\
\hline Inclined orbit constellation & $N$ & $i / \mathrm{deg}$ & $n_{1}$ & $n_{2}$ & $\min / \mathrm{deg}$ \\
\hline Symmetrical polar constellation & 1073 & 55.1 & 29 & 37 & 5.06 \\
Non-symmetrical polar constellation & 522 & 90 & 17 & 39 & 5.29 \\
\hline
\end{tabular}

All the analyses above are based on specified single coverage performance. Then the effect of single coverage angle $\theta$ is investigated by changing the orbit altitude. The required satellite numbers of the three kinds of constellations are calculated at different orbit altitudes. As Figure 12 shows, 
because of the growth of $\theta$ by increasing orbit altitude, both the required $n_{1}$ and $n_{2}$ decrease, which results in the decrease of corresponding total number of satellites. Comparing the three kinds of constellations, one can find that non-symmetrical constellation requires the least satellites to achieve the same coverage. The detailed parameters of the optimal constellations at different altitudes are listed in Table 4. It can be found that required satellites in inclined orbit constellations are approximately twice the number of non-symmetrical polar constellations.

Note that only the coverage of street region is considered in SOC method, thus the extra coverage beyond the streets is neglected in the design of inclined orbit constellations. Then the number of satellite can be further reduced when the extra coverage is exploited. However, the analysis will be much more complicated. The sufficiently covered results in this paper will provide theoretical guidance and preliminary reference for constellation design.

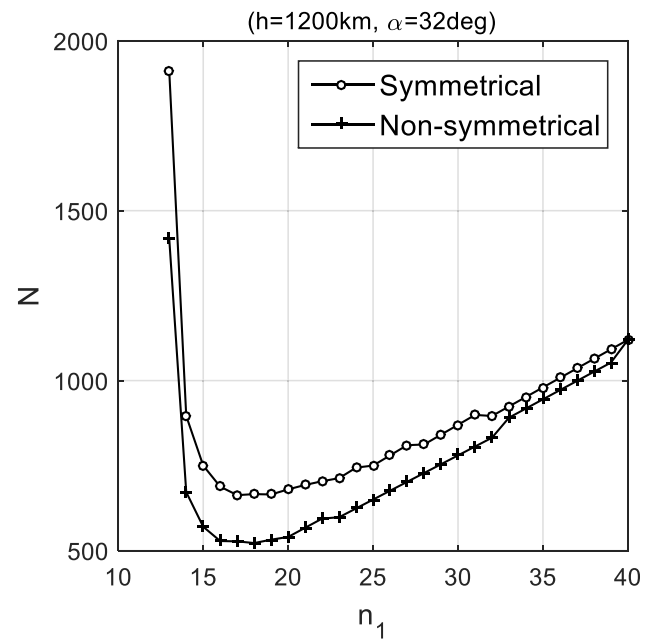

Figure 11. Number of satellites of polar constellations.

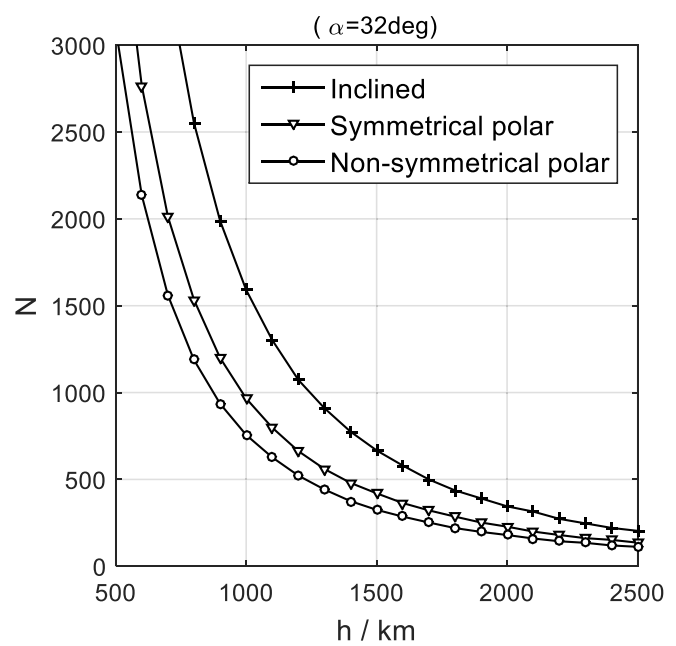

Figure 12. Satellite number at different altitudes.

Table 4. Optimal constellation configurations at different orbit altitudes.

\begin{tabular}{|c|c|c|c|c|c|c|c|c|c|}
\hline \multirow[b]{2}{*}{$h / \mathrm{km}$} & \multirow[b]{2}{*}{$\theta /$ deg } & \multicolumn{4}{|c|}{ Inclined orbit constellation } & \multicolumn{3}{|c|}{$\begin{array}{c}\text { Non-symmetrical Polar } \\
\text { constellation }\end{array}$} & \multirow[b]{2}{*}{$N_{\text {polar }} / N_{\text {inclined }}$} \\
\hline & & $N$ & $n_{1}$ & $n_{2}$ & $i / \mathrm{deg}$ & $N$ & $n_{1}$ & $n_{2}$ & \\
\hline 500 & 2.86 & 6860 & 70 & 98 & 57.90 & 3096 & 43 & 72 & 0.451 \\
\hline 600 & 3.44 & 4636 & 61 & 76 & 57.60 & 2135 & 35 & 61 & 0.461 \\
\hline 700 & 4.03 & 3366 & 51 & 66 & 57.10 & 1560 & 30 & 52 & 0.463 \\
\hline 800 & 4.62 & 2552 & 44 & 58 & 56.70 & 1188 & 27 & 44 & 0.466 \\
\hline 900 & 5.21 & 1989 & 39 & 51 & 56.30 & 936 & 24 & 39 & 0.471 \\
\hline 1000 & 5.81 & 1591 & 37 & 43 & 56.10 & 756 & 21 & 36 & 0.475 \\
\hline 1200 & 7.03 & 1073 & 29 & 37 & 55.10 & 522 & 18 & 29 & 0.486 \\
\hline 1500 & 8.90 & 667 & 23 & 29 & 53.90 & 325 & 13 & 25 & 0.487 \\
\hline 2000 & 12.13 & 345 & 15 & 23 & 51.20 & 180 & 10 & 18 & 0.522 \\
\hline 2500 & 15.55 & 204 & 12 & 17 & 49.40 & 112 & 8 & 14 & 0.549 \\
\hline
\end{tabular}

\section{Conclusion}

In this paper, Streets-of-Coverage method is applied to design inclined and polar orbit constellations providing continuous coverage of mid-and low-latitude zonal regions under the constraint of prescribed half-beam angle. The results indicate that single coverage angle is the core factor that influences the constellation configuration and corresponding number of satellite. When narrowbeamwidth antenna is adopted to provide internet services, up to thousands of LEO satellites are required to continuously cover mid-and low-latitude regions due to the small single coverage. 
When the single coverage performance is specified, inclined orbit constellation is mainly affected by inclination and the number of orbital planes. The inclination of the optimal result is close to the highest latitude to be covered while slightly lower than it. If the inclination is fixed, the required satellite firstly decreases then increases with the increment of orbit planes. Compared to inclined orbit constellations, polar constellations are able to save half of the satellites to provide the same coverage because of the less overlapped regions between two adjacent planes. When the phase difference and coverage beyond streets are considered, non-symmetrical configuration is better than symmetrical case. In the case of $h=1200 \mathrm{~km}, \alpha=32^{\circ}$, the required number of satellites of inclined orbit, symmetrical polar and non-symmetrical polar cases are 1073, 663 and 522 respectively. Increase of orbital altitude and satellite scale are the two effective methods to improve continuous coverage performance.

\section{Acknowledgment}

This work was supported by the National Natural Science Foundation of China (61302092) and Preresearch Project of National University of Defense Technology (ZDYYJCYJ20140701).

\section{References}

1. D.J. Barnhart, T. Vladimirova, M.N. Sweeting, Very-small-satellite design for distributed space missions, J Spacecr Rockets, 44, 1294-1306 (2007)

2. Home - OneWeb, http://oneweb.world/\#solution (Accessed 14 November 2016)

3. SpaceX satellite constellation, http://en.wikipedia.org/wiki/SpaceX_satellite_constellation (Accessed 14 November 2016)

4. G. Maral, J.J.d Ridder, B.G. Evans, M. Richharia, Low earth orbit satellite systems for communications, Int J Satell Commun Network, 9, 209-225 (1991)

5. G. Maral, M. Bousquet, Satellite Communications Systems: Systems, Techniques and Technology, (John Wiley \& Sons, Chichester, 2010)

6. M. Ibnkahla, Q.M. Rahman, A.I. Sulyman, H.A. Al-Asady, Y. Jun, A. Safwat, High-speed satellite mobile communications: technologies and challenges, Proc IEEE, 92, 312-339 (2004)

7. R.D. Luders, L.J. Ginsberg, Continuous zonal coverage: a generalized analysis, AIAA Mechanics and Control of Flight Conference, NO 74-842 (1974)

8. D. Palombini, S. Arena, T. Cavanna, E. Limiti, Compact sub-harmonic mixer for Q-band satellite communications, 2014 9th European Microwave Integrated Circuit Conference, 61-64 (2014) 